\title{
LA SOMIGLIANZA ICONICA NELLA SCRITTURA DE-SOGGETTIVANTE TRA IDENTITÀ E DIFFERENZA
}

\section{ICONIC SIMILARITY IN THE DE-SUBJECTIVE WRITING BETWEEN IDENTITY AND DIFFERENCE}

\author{
Luciano PONZIO \\ Università del Salento-Lecce \\ luciano.ponzio@unisalento.it
}

Riassunto: Il pericoloso potere di assimilazione renderebbe le cose identiche le une alle altre, uniformandole, smarrendone la singolarità. Il mondo senza alterità si ridurrebbe a una massa omogenea: l'Identico e la sua rappresentazione. L'esistenza di un segno non è semplicemente riflessa ma rifratta (Vološinov). In questa rifrazione gioca il ruolo determinante l'alterità. Produrre e comprendere segni significa partecipare ai processi comunicativi. Il segnico è il campo dell'ambivalenza in cui tutto si decide per relazioni e pratiche sociali (Bachtin). Uscire dalla filosofia del soggetto (Foucault), porsi in una posizione "fuori-di-sé", configurare una scrittura in-ferma contro il soggetto statico, per recuperare il corpo sociale nelle parole e nelle immagini.

Parole chiave: Similarità. Scrittura. Alterità. Intercorporeità. Iconicità.

Abstract: The dangerous power of assimilation uniforms things, making them lose their singularity. The world without otherness would be reduced to a homogeneous mass where representation always represents the Identical. The existence of a sign is not simply reflected but refracted (Voloshinov). In this refraction, otherness plays the decisive role. Producing and understanding signs means participating in communication processes. The sign is the field of ambivalence in which everything is decided by relationships and social practices (Bakhtin). In this field is possible as this paper tries to show, to get out of the philosophy of the subject (Foucault) in order to recover the social body of words and images. 
Key Words: Similarity. Writings. Otherness. Intercorporeity. Iconicity.

Dal punto di vista semiotico, lo studio della produzione e dei processi della comunicazione sociale evidenziano l'inseparabilità tra segno e ideologia. L'esistenza di un segno non è semplicemente riflessa ma rifratta (Vološinov, 1929; vedi anche Vološinov 1926 e 1930). In questa rifrazione gioca il ruolo determinante l'alterità.

La produzione di un segno non è mero processo di identificazione; $\mathrm{i}$ segni non sono dati una volta e per tutte. Il pericoloso potere di assimilazione, renderebbe le cose identiche le une alle altre, uniformandole, facendone smarrire la singolarità. Il mondo senza alterità si ridurrebbe a una massa omogenea, alla smorta figura del Medesimo, dell'identico e della sua rappresentazione, ripetizione, imitazione.

Ma l'antipatia dell'Altro ne impedisce l'assimilazione.

Produrre e comprendere segni significa partecipare ai processi comunicativi. Il segnico è il campo dell'ambivalenza, della somiglianza, della deviazione, della creatività, in cui tutto si decide per relazioni e pratiche sociali (io-per-me, l'altro-per-me e io-per-l'altro: in Bachtin e il suo Circolo, 2014: 129).

Il testo artistico fornisce la possibilità di cogliere al meglio la struttura dialogica dell'enunciazione.

Nella terminologia di Bachtin riferita all'accentuazione ovvero all'orientamento dell'enunciazione, intesa come cellula viva del parlare, e a proposito della quale egli parla di risonanza, o alla caratteristica di un certo tipo del genere romanzo indicato come polifonico, non ci sono separazioni tra le sfere della produzione dei testi che ne impediscano l'attraversamento e il rinvio alle caratteristiche di una di esse per comprendere le caratteristiche di un'altra: "Sono l'interrelazione fra i generi primari e secondari e il processo di formazione storica di questi ultimi a gettare luce sulla natura dell'enunciazione (interrelazione fra lingua e ideologia, la concezione del mondo)" (Bachtin, 1979b: 247-248). Bachtin mostra come il senso del testo non dipenda dagli elementi ripetibili del sistema di segni con cui è composto (interpretanti di identificazione) ma nella sua stessa costituzione, e non solo nella sua manifestazione, esso è situato nei rapporti di rinvio, di differimento (interpretanti di comprensione rispondente) che danno luogo 
ad una catena di testi ad esso antecedenti e ad esso successivi, rientranti in una certa sfera, quella relativa al tema trattato, al genere testuale, ad un determinato settore disciplinare. Vološinov (1929) definisce l'enunciazione un "contesto di vita" più o meno ampio, potenziale patrimonio sociale della vita umana.

Non esiste dunque un segno solo, un testo isolato, unico. Un segno ha bisogno di un altro segno (interpretante) per essere segno. Affinché ci sia un segno ce ne vuole un altro, e quindi un altro, e così via.

L'enunciazione è realizzabile solo in uno spazio dialogico. E tale dialogicità si realizza unicamente in una relazione intercorporea. La scrittura letteraria ha ampiamente dimostrato questa dialogicità nelle forme miste di riportare il discorso, ascoltando voci e considerando centri di valore differenti. Il discorso indiretto libero (variante dei discorsi assoggettanti indiretto e diretto) è una di queste strategie letterarie emancipanti per far sì che un testo sia artistico, dialogico, ottuso (Barthes, 1982) e enunciazione non iterabile. La scrittura letteraria è una scrittura pittorica non sempre lineare, se consideriamo, come ci insegna Bachtin (Bachtin-Vološinov, 1929), la differenza tra "stile lineare" e "stile pittoresco", quest'ultimo capace di indebolire i contorni rigidi di corpi chiusi e separati. Tale indebolimento non avviene nella forma "retorica", dove si esige una percezione ancora chiara delle frontiere tra discorso proprio e parola altra, ma appunto nel discorso indiretto libero. Nel dispiegarne la trama, pur rispettandone regole, forme e strutture, l'enunciazione letteraria del discorso indiretto libero è capace di trasgredirle in un fitto dialogo.

L'enunciazione può avvenire sia nel testo verbale che nel testo non verbale. L'autoritratto, per esempio, è un genere testuale che si presta bene a evidenziare questo (paradossale) luogo di non rappresentazione, di raffigurazione "non lineare" ma "pittoresca", figurale (in contrapposizione alla figurazione, illustrazione, come direbbe su Bacon Deleuze (1985).

Considerando il termine auto-ritratto come ritrar-si, ritrattar-si, fino mal-trattarsi - il termine esecuzione significa non solo mettere in opera ma anche l'atto crudele (Artaud, 1938) di messa a morte, di distruzione: cioè eliminare l'io, il medesimo, collocandosi il più lontano possibile da se stessi, dando vita a un linguaggio "fuori-di-sé", "fuori dal significare per sé" (Derrida, 1986), rivelando uno scarto più che un ripiegamento, una dispersione più che un ritorno dei segni su loro stessi-, pur di distruggere l'immagine riflessa come specchio, mimesi, immagine rappresentativa 
e rappresentazionale, posa. Nell'autoritratto l'immagine non è "posa" (come invece il comando d'esecuzione fotografico impone per mortificare l'oggetto inquadrato) e non "ri-posa" mostrando sviluppi che la fotografia non possiede (almeno all'inizio, soprattutto un certo tipo di fotografia legata e impiegata alla riproduzione dell'identità). La fotografia si sviluppa, pertanto, senza ulteriore "sviluppo".

L'autoritratto come autoritrarsi, trarsi fuori, vedersi come altro (Derrida, 1990; tr. it., 2003; vedi a questo proposito anche Nancy, 2002 e 2014), è prima di tutto autoritratto dell'altro, qui nel doppio movimento tensionale, vale a dire: "l'altro ritratto" significa sia "l'altro che viene riprodotto" sia "l'altro che si ritrae dall'essere ritratto". In questa tensione del ritrarsi, autore-creatore ed autore-uomo non coincidono, bensì autorecreatore ed eroe. L'autoritratto è il paradigma del testo artistico bifronte non costretto a scelte oppositive: oggettiva/soggettiva, significato/ significante, figura/sfondo, forma/contenuto. L'autoritratto è un continuo attentato al principio di identità. L'autoritratto è ciò che non aderisce all'immagine sé-dicente, che dice se stessa (e crede di dire), né ripiega sul verbo essere, ma rinvia e rilancia il senso nella direzione di un'alterità non relativa ma assoluta, laddove l'altro è l'inaspettabile: ciò o colui che non ti aspetti, in un incontro-evento, per apparizione, un'apparizione non più legata al mondo delle apparenze, e in esso si rivela la sua inestricabilità, ineliminabililità. L'arte è questo movimento arrischiato, dove l'incontro con l'altro è il tratto-rischio dell'arte (Derrida, 2010).

L'autoritratto rinvia l'autore stesso a "vedersi" come altro, e non come identico. La sua immagine non aderisce punto per punto con l'immagine "reale", "realistica", "ufficiale" ma di essa considera solo alcuni punti, alterandone dettagli, particolari che la caratterizzano e la caricaturizzano in una immagine che si fa immagine, raffigurazione (Bachtin izobrazenie: obraz = immagine), una somiglianza iconica differita, non più concepita nella forma di immagine-idolo come, filiazione di identità e differenza.

L'autoritratto è, pertanto, un genere e un testo estremamente dialogico, laddove l'alterità si manifesta in tutte le sue gamme espressive. Qui l'autore si colloca necessariamente in una situazione di ascolto come altro di sé (mostrando come vorrebbe essere a se stesso), e con l'altro fuori di se (come vorrebbe mostrarsi agli altri ma anche come gli altri vedrebbero lui stesso). 
Tornando alla parola letteraria, alla letteratura, meglio alla scrittura letteraria che ha ormai acquisito, come dice Michel Foucault, "il diritto di uccidere, di essere l'assassina anche del suo autore a cui spetta il ruolo del 'morto' nel gioco della scrittura" (Foucault, 1994: 4; 1984), un certo affrancamento dall'autore/autoritario (già prima regola dei cosiddetti formalisti russi, nonché dell' architettonica estetica di Bachtin - "l'autoreuomo deve indossare la veste del tacere"-, nonostante la sua critica al formalismo).

Come negazione del ruolo della soggettività, Michel Foucault, in Le parole e le cose (1966; tr. it., 2001), originariamente intitolato L'ordine delle cose, usando come pretesto le avventure di Don Chisciotte (III parte, intitolata Rappresentare) in relazione alla somiglianza e ai segni, descrive l'eroe di Cervantes.

Don Chisciotte è fatto a immagine e somiglianza dei segni: "lungo grafismo magro come una lettera, eccolo emerso direttamente dallo sbadiglio dei libri”, scrive Foucault (1966; tr. it., 2001: 61). L'intero suo essere non è che linguaggio, testo, fogli stampati, storia già scritta. Don Chisciotte è fatto di parole intersecate, è scrittura errante nel mondo in mezzo alla somiglianza delle cose: è "l'eroe del Medesimo" nelle "provincie dell'Analogo".

Il libro è così più il suo dovere che la sua esistenza, e Don Chisciotte, senza tregua, deve continuamente consultarlo per sapere cosa fare e cosa dire e quali segni dare a se stesso e agli altri per mostrare che la sua natura è la stessa del testo dal quale è uscito. Don Chisciotte, dice Foucault, acquisisce la sua realtà interna alle parole, non nel rapporto di similitudine tra parole e mondo, ma nella relazione che i segni verbali intrecciano da sé a sé, laddove le parole ripiegano su sé stesse per analogia. Non si avrà più a che fare con similitudini, ma con identità e differenze, avverte Foucault. Sicché chi coglie ancora similitudini e segni, è il pazzo, Il Differente per eccellenza, nella misura in cui non conosce le differenze, "vede ovunque somiglianze e segni della somiglianza": "tutti i segni si somigliano e tutte le somiglianze valgono come segni" (1966; tr. it., 2001: 64), chi non conosce la differenza viene a costituire la devianza dell'ordine convenuto. Il poeta (associato al folle) è colui che ritrova queste similitudini disperse sepolte nelle cose, sotto la Sovranità del Medesimo.

Le parole trovano il loro potere di estraneità ed è la Somiglianza che si colloca dalla parte della fantasia e l'iconicità nell'accezione di Peirce 
si basa proprio sul rapporto di somiglianza, ed è la parte più creativa del segno (1931-58; 1980; 2003).

Don Chisciotte insegue il corpo quotidiano, ma la scrittura letteraria impone alla parola la cerimonia del carnevale, che ne fa di essa corpo grottesco, glorioso e insieme scomparsa del corpo visibile, possibile, dicibile, rappresentabile, autosufficiente, sé-dicente e chiuso.

Al fianco dell'infunzionale alterità della scrittura, alla sua non ufficialità, ostile a qualsiasi compiutezza, stabilità, determinatezza del pensiero e della concezione del mondo (Barthes 1973), si colloca la somiglianza iconica che impone alla parola e alle immagini la cerimonia del carnevale, che scrive nel segno dell'altro, del dire della sua persistenza, irriducibile ad una identità, della sua ineliminabilità, che ne è vera la cifra, la singolarità, l'unicità, di questa assoluta alterità.

La scrittura de-soggettivante strappa il soggetto (sempre identico a se stesso) a se stesso: "strapparmi' a me stesso" — dice Foucault (in Trombadori, 2005: 30-32) - "impedendomi di essere sempre lo stesso", facendo in modo che non sia più tale, o che sia completamente altro da sé "uscendone trasformati", stabilendo, a conclusione del libro, nuovi rapporti con l'eroe, vale a dire con ciò di cui ci si intrattiene scrivendo.

Bachtin recupera in corpo sociale fatto di parole e immagini nella festa popolare del suo Rabelais. Il corpo grottesco, come ci dice Bachtin (1965) su Rabelais, è schierato contro ogni assoggettamento e rappresentazione, contro il potere e il suo esercizio di imporre identità e differenze:

Il carnevale è uno spettacolo senza ribalta e senza divisione in esecutori e spettatori. Nel carnevale tutti sono attivi partecipanti, tutti prendono parte all'azione carnevalesca. Il carnevale non si contempla e non si recita, si vive in esso, si vive secondo le sue leggi, finché queste leggi sono in vigore, cioè si vive la vita carnevalesca. Ma la vita carnevalesca è una vita tolta al suo normale binario, è in una certa misura una "vita all'incontrario", un “mondo alla rovescia" (Bachtin, 1929; tr. it., 1963: 160).

Scritto nel corso della seconda guerra mondiale, il lavoro di dissertazione su Rabelais, risultò talmente rivoluzionario che a Bachtin venne negato il dottorato (il libro fu pubblicato solo nel 1965). I corpi di parole e i corpi di immagini prodotti nel Rabelais sono la cifra della 
concezione artistica non-letteraria, non conforme ai canoni, alle regole "letterarie". La sua non-ufficialità è ostile a qualsiasi compiutezza e stabilità, serietà angusta, finitezza, determinatezza del pensiero e della concezione del mondo.

Il recupero del carnevalesco nei riti e culti comici, nelle immagini di buffoni, stolti, giganti, nani, mostri, giullari dà luogo a una letteratura parodica dei cerimoniali seri della rappresentazione. Il senso marcatamente non-ufficiale affianca il mondo ufficiale, redendo visibile un secondo e molteplice mondo, una seconda vita, un dualismo del mondo che duplica l'aspetto del mondo, percezione della vita. Tale visione mette insieme e rimette in gioco i culti seri con i culti comici, ugualmente reali, in una immagine del mondo celebrata e derisa insieme. Il principio di carnevalizzazione libera i rituali sociali da ogni dogmatismo, e l'elemento del gioco si rivela determinante nelle forme artistiche e raffigurative.

Creare uno spazio testuale (si veda Per una filosofia dell'atto responsabile, in Bachtin, 1920-24; tr. it. in Bachtin e il suo Circolo, 2014: 33-167) non significa allargare l'orizzonte della scrittura solo sul piano "letterale" ma anche e soprattutto in considerazione del valore estetico di un'opera (misurabile come letterarietà), di un tratto scrivente che ci permetterebbe finalmente di vedere, di ascoltare la parola altra.

La parola e la sua funzione sociale si oppongono, dunque, al soggetto statico, e sono soprattutto le parole e le immagini della raffigurazione artistica a recuperare il corpo sociale, in una connaturata intercorporeità.

La sfida che lancia Bachtin è proprio quella di portare nella vita ciò che si scopre nell'arte. In questo senso, l'atto di resistenza nella scrittura letteraria ("scrivere per non morire", Blanchot; "l'arte è ciò che resite alla morte", Deleuze) ci permette di ri-esistere, di rivivere una seconda vita (non ufficiale ma ugualmente possibile) e di rinascere nella visione artistica (il testo artistico non vuol essere altro che questo spazio di sperimentazione e sistema di modellazione di nuovi mondi possibili).

L'elogio del "mondo alla rovescia" (Bachtin) e del "corpo grottesco" rimanda al "corpo senza organi" di cui parla per primo Artaud, in un esperimento non solo radiofonico ma "biologico" (diranno Deleuze e Guattari, 1980). Il corpo senza organi non si oppone agli organi ma si oppone all'organismo (Deleuze e Guattari, 1980), all'organizzazione organica degli organismi che impongono al corpo forme, funzioni, collegamenti, organizzazioni dominanti e gerarchizzate, organizzate per 
estrarne un lavoro utile. liberato da tutti gli automatismi è restituito alla sua vera e illimitata libertà — dice Artaud (2001, 2003) — re-insegnando al corpo a danzare alla rovescia (come nel delirio ritmico del bal-musette), e questo rovescio sarà il suo vero diritto.

In attesa che l'opera sovrasti il suo autore fino a liberarsene (la separazione dell'opera dal suo autore costituisce la prima regola del formalismo, ripresa anche da un Bachtin "formalista"), l'opera stessa mostra già tutta la sua alterità nella scrittura letteraria (qui con valore di letterarietà e che possiamo ritrovare non esclusivamente nella Letteratura). Una scrittura letteraria che, nella sua paradossale illegibilità e inconsumabilità, si mostra come atto di resistenza: scrivere è resistere. Che cos'è la scrittura? — si domanda Barthes (1998: 287)—. La scrittura è ciò che resiste alla Lettura, ciò che non si lascia leggere una volta e per tutte.

La rinascita nell'arte è nella memoria impossibile di Chagall, in Ma Vie, dove egli ricorda di esser nato morto e rinato nell'incontro con la pittura. È quanto succede a Barthes (1980), occupandosi del testo fotografico, nella ricerca dell'immagine di sua madre dopo la morte di lei, ritrovandola nella scrittura, in una memoria impossibile, un'immagine cronologicamente non direttamente vissuta, in un rapporto di somiglianza, "iconico", che Barthes definisce "essenza" (ivi). Laddove l'universo fisico si ferma con la morte, la scrittura letteraria, l'arte, resiste. In una condizione di isolamento, tra scrittura e morte, "scrivere è vivere", sosteneva Kafka ricordato da Foucault. "L'autore deve recitare il ruolo di morto nel gioco della scrittura", scrive proprio Foucault (1994: 4); "l'autore-uomo deve la veste del tacere", dice Bachtin (1979).

La scrittura letteraria è un linguaggio all'infinito rivela Foucault (1994: 73-85) in cui Le Mille e una notte è solo un esempio di "cronotopo letterario" in grado di sospendere per pagine e pagine il fluire inesorabile del tempo relativo ("cronotopo quotidiano"): qui quel che conta per non morire è il racconto di una scrittura interminabile (Foucault nel suo testo sopra menzionato fa riferimento anche a Il miracolo segreto di Borges, e al protagonista, l'ebreo Hledík, anche lui scrittore che vince la morte).

Jakobson (1978: 18) insiste particolarmente sul ruolo centrale della funzione poetica nello studio del linguaggio: la funzione poetica è all'opera in ogni espressione verbale, è una delle funzioni essenziali del linguaggio, che appare necessariamente nella parola, in qualsiasi enunciazione, cellula viva del discorso: "Senza la funzione poetica, il linguaggio è morto. 
L'attività dei segni verbali scompare. Il linguaggio diviene del tutto statico. La funzione poetica introduce un dinamismo nella vita del linguaggio" (Jakobson, 1978: 18). Il senso dell'enunciazione non risiede nella letteralità della prosa, nella dimensione frastica del significato, ma nella letterarietà (qui, come detto, non in senso stretto, riservato alla letteratura).

Attento studioso del linguaggio poetico, Jakobson individua, nell'opera di Majakovskij, alcuni temi ricorrenti (l'amore, il suicidio, il gioco, la morte, la resurrezione, l'irrazionale) e li riorganizza in una sorta di simbologia.

Sulle capacità abduttive e anticipatrici di Majakovskij, Jakobson si sofferma sui dettagli sparpagliati nei suoi innumerevoli poemi, come vere e proprie profezie che si realizzarono "alla lettera". Un legame indissolubile tra vita e creazione artistica. La metafora del gioco, "Majakovskij visse e morì da giocatore", dice Jakobson (1989: 132), mostra ancora una volta il carattere arrischiato tipico della scrittura letteraria, ma allo stesso tempo delinea quasi un progetto del poeta e ne configura la sceneggiatura in cui "la posta in gioco è la vita stessa del poeta".

Morte, scrittura, alterità e resistenza si ritrovano nel linguaggio poetico. Nel segno della resurrezione (La resurrezione della parola, così titolava uno dei primi scritti a firma di Šklovskij (1914; tr. it. in Prevignano, a cura, 1979: 101-108; v. anche in Ferrari-Bravo e Treu, 2010: 119-127); anche l'eroe di La cimice di Majakovskij, Prisypkin, ripropone il tema della "resurrezione"), dice Jakobson, Majakovskij supera e sprona il tempo ("si tratta di un antico principio di Majakovskij: "il poeta deve affrettare il tempo"". "Ed ecco che egli guarda le proprie righe, scritte poco prima della morte, con gli occhi del lettore di dopodomani" (Jakobson, 1930, tr. it., 2004: 15), affascinato dalle nuove scoperte scientifiche per raggiungere l'immortalità e il sogno dell' "eterno terrestre" ("Non ho vissuto sino in fondo la mia vita terrena // sulla terra / non ho avuto tutto il mio amore... Resuscitatemi: voglio vivere tutta la mia vita!" (Jakobson, 1972; tr. it., 1989: 136), tema dell'eterno terrestre "violentemente contrapposto a ogni sorta di incorporea astrazione sovramondana" (Jakobson, tr. it., 2004: 36).

Nell'opera di Majakovskij, Jakobson associa all'"Io creativo", un'alterità, un antagonista, un "non io", "un orribile sosia" il cui "credo è la stabilizzazione e l'autoisolamento": 1'Io della vita quotidiana, è dipinto come un "possessore-accumulatore, che Chlebnikov contrappone all'inventore" (Jacobson, 1989: 21-22). È lotta contro la rappresentazione 
quotidiana, "incrollabile ordine universale", di un universo che "vive isolato in appartamenti", che opprime la vita del poeta, dice ancora Jakobson, e dove "tutto è muto". Jakobson riprende diverse formule-immagini del poeta e amico Majakovskij, che prova in tutte le maniere di liberarsi del "nemico di sempre", quell'essere (byt) statico delle cose, "la vita quotidiana senza movimento", "lo stagno dell'esistenza quotidiana [...] riempito di fanghiglia e [...] coperto delle erbe palustri della monotonia" (Jacobson, 1989: 19).

L'affermazione dell'irrazionale è in Majakovskij l'amore (L'amore è il cuore di tutte le cose), schiacciato dalla vita quotidiana e dalla sonnolenta noia della banalità. Un amore che si vendica di chi ha osato dimenticarlo (Jakobson, 1989: 27). La brama di conoscere la propria vita, di anticiparne il proprio destino, costante consapevolezza dell'irrimediabilità della fine, porterà Majakovskij dal verso alla prosa, tanto che il tema del suicidio si è talmente avvicinato che non è più possibile raffigurarlo, laddove la metafora si fa metonimia.

Jakobson perviene alla conclusione della sua tesi sull'opera di Majakovskij come "montaggio di immagini" di una scrittura anticipatrice: "Sembra che mai il destino dello scrittore sia stato messo a nudo attraverso le sue stesse parole con tanta impietosa franchezza come ai nostri giorni. Egli brama conoscere la propria vita in anticipo e la riconosce nel suo proprio romanzo" (Jacobson, 1989: 39). Un addio già pronto da tempo, definito da Jakobson: un laboratorio sulla parola portato a perfezione (La barca dell'amore si è spezzata contro la vita quotidiana). Tanto si passa dal verso alla prosa, che "l'ultima morte" (un duello fino all'"ultima morte" - lo stesso Majakovskij, scrive Jakobson $(1989 ; 52)$ "paragonava il proprio duello con la vita quotidiana ai duelli di Puškin e Lermontov", fino a quando "tema del suicidio" si era "talmente avvicinato" che non fu più possibile "raffigurarlo" (Jacobson, 1989: 39 e 41-42), "unione tra l'ineluttabile 'ultima morte' ('Ma non ho scampo') e il volontario 'fare i conti con la vita' si rivelò a Majakovskij e si decantò in parola e in azione nel fatale aprile del 1930" (Jakobson, 1989: 153).

"Parole, proprie e altrui, convivevano in Majakovskij con una costanza sorprendente" (Jakobson, tr. it. 1989: 140). Tracciare oggi un confine tra la mitologia poetica e il curriculum vitae dell'autore non è possibile: nella vita del poeta conta ciò che si è depositato nei versi. Leggere Majakovskij, dice Jakobson, significa considerare gli effetti della 
sua poesia verso il futuro, e non risalire alle cause.

\section{RIFERIMENTI BIBLIOGRAFICI}

ARTAUD, A. (1938) [2000]. Il teatro e il suo doppio, pref. di J. Derrida, a cura di G. R. Morisco e G. Neri. Torino: Einaudi. (2001). Per farla finita col giudizio di Dio, M. Dotti (ed.). Roma: Stampa Alternativa. (2003). CsO: Il corpo senz'organi, M. Dotti (ed.). Milano: Mimesis. BACHTIN, M. M. (1920-24) [2014]. "K filosofii postupka". ["Per una filosofia dell'atto", tr. it. in M. M. Bachtin e Il suo Circolo, Opere 1919-1930, 26-167. Milano: Bompiani.]

(1929) [2014]. "Problemy tvorčestva Dostoevskogo". Leningrado: Priboj. ["Problemi dell'opera di Dostoevskij", tr. it. in M. M. Bachtin e Il suo Circolo, Opere 1919-1930, 1052-1423. Milano: Bompiani.]

(1952-53) [1979]. "Il problema dei generi del discorso". In L'autore e l'eroe, M. M. Bachtin, 245-290. Torino: Einaudi.

(1963) [2002]. Problemy poetiki Dostoevskogo. Mosca: Sovetskij pisatel. [Problemi della poetica di Dostoevskij, tr. it. di G. Garritano, M. M. Bachtin, Dostoevskij. Poetica e stilistica. Torino: Einaudi.] (1965) [1979]. Tvorčestvo Fransua Rable i narodnaja kul'tura srednevekov'ja i Renessansa. Mosca: Izdatel'stvo "Chudožestvennaja literatura". [L'opera di François Rabelais e la cultura popolare nel Medioevo e del Rinascimento, tr. it. di M. Romano. Torino: Einaudi.]

(1979a). Estetika slovesnogo tvorčestva. Mosca: Izdatel'stvo "Iskusstvo". [Estetica della creazione verbale, tr. it. di C. Strada Janovič, M. M. Bachtin, L'autore e l'eroe. Teoria letteraria e scienze umane. Torino, Einaudi.]

(1979b). L'autore e l'eroe. Teoria letteraria e scienze umane. Torino: Einaudi.

(1979c). Estetica e romanzo. Torino: Einaudi.

(1979d). "Il problema del testo nella linguistica, nella filologia e 
nelle altre scienze umane: una analisi filosofica". In L'autore e l' eroe. Teoria letteraria e scienze umane, M. M. Bachtin, 291-329. Torino: Einaudi.

BACHTIN, M. e IL SUO CIRCOLO (2014). Opere 1919-1930. Milano: Bompiani.

BARTHES, R. (1973). Les plaisir du texte. Paris: Seuil [tr. it. Variazioni sulla scrittura (1994) seguite da Il piacere del testo, a cura di C. Ossola. Torino: Einaudi, 1999].

(1980). La camera chiara, Torino: Einaudi.

(1982). L'obvie et l'obtus. Essais critiques III. Paris: Seuil. [Trad. it. di C. Benincasa, G. Bottiroli, G. P. Caprettini, D. De Agostini, L. Lonzi, G. Mariotti, L'ovvio e l'ottuso. Saggi critici III. Torino: Einaudi, 1985].

(1998). Scritti. Società, testo, comunicazione, G. Marrone (ed.). Torino: Einaudi.

DELEUZE, G. (1985). Francis Bacon. Logica della sensazione. Macerata: Quodlibet.

DELEUZE, G. e GUATTARI, F. (1980). Millepiani. Capitalismo e schizofrenia, M. Carboni (ed.). Roma: Castelvecchi.

DERRIDA, J. (1986) [2005]. Antonin Artaud. Forsennare il soggettile. Milano: Abscondita.

(1990) [2003]. Memorie di cieco. L'autoritratto e alter rovine. Roma:

Newton Compton.

(2010). Pensare al non vedere. Scritti sulle arti del visibile (19792004). Milano: Jaka book.

FERRARI-BRAVO, D. e TREU, E. (2010). La parola nella cultura russa tra '800 e '900. Pisa: Tipografia Pisana.

FOUCAULT, M. (1966) [1998]. Le parole e le cose. Un'archeologia delle scienze umane. Milano: Rizzoli.

(1984a). "What is an Author?”. In The Foucault Reader, P. Rabinow (ed.), 101-120. New York: Pantheon.

(1994). Scritti letterari. A cura di Cesare Milanese. Milano: Feltrinelli.

JAKOBSON, R. (1931) [2004]. O pokolenij, rastrativ̌̌em svoich poetov. [Tr. it. di V. Strada (ed.), Una generazione che ha dissipato i suoi poeti. Milano: SE.] 
(1978). “Entretien”. Cahiers du Cistre 5, 11-26.

(1989). Russia, follia, poesia. Napoli: Guida.

NANCY, J. L. (2002). Il ritratto e il suo sguardo. Milano: Cortina. (2014). L'altro ritratto. Roma: Castelvecchi.

PEIRCE, C. S. (1931-58). Collected Papers, 8 vols. Cambridge, MA: Harvard University Press.

(1980). Semiotica. Torino: Einaudi. (2003). Opere. Milano: Bompiani.

PREVIGNANO, C. (1979). La semiotica nei paesi slavi. Milano: Feltrinelli.

TROMBADORI, D. (2005). Colloqui con Foucault. Pensieri, opere, omissioni dell'ultimo maître-à-penser. Roma: Castelvecchi.

VOLOŠINOV, V. N. (1926) [2014]. "Slovo v žizni i slovo v poezii". Žvezda 6, 144-167. [La parola nella vita e la parola nella poesia, tr. it. con testo russo a fronte in M.M. Bachtin e Il suo Circolo, Opere 1919-1930, 270-333. Milano: Bompiani.]

(1929) [2014]. Marksizm i filosofja jazika. Leningrado: Priboj. [Marxismo e filosofia del linguaggio, tr. it., in M.M. Bachtin e Il suo Circolo, Opere 1919-1930, 1458-1839. Milano: Bompiani.]

(1930) [2014]. "Stilistika chudožestvennoj reči”. Žvezda, 2, 48-66; 3, 65-87; 5, 43-59. [Stilistica del discorso artistico, tr. it. con testo russo a fronte in M.M. Bachtin e Il suo Circolo, Opere 1919-1930, 1841-1993. Milano: Bompiani.]

VOLOŠINOV, V. N. e BACHTIN, M. M. (1929) [2010]. Parola propria e parola altrui nella sintassi dell'enunciazione. Lecce: Pensa MultiMedia.

Recibido el 2 de marzo de 2020.

Aceptado el 15 de abril de 2020. 